\title{
The role of extracellular matrix metalloproteinases and their inhibitors in allergic diseases
}

\author{
Andrzej Kuźmiński, Michał Przybyszewski, Małgorzata Graczyk, Zbigniew Bartuzi
}

Department of Allergology, Clinical Immunology and Internal Diseases, Nicholas Copernicus University in Torun, Ludwik Rydygier Collegium Medicum in Bydgoszcz, Poland

Head: Prof. Zbigniew Bartuzi MD, PhD

Postep Derm Alergol 2012; XXIX, 5: 384-389

DOI: 10.5114/pdia.2012.31493

\begin{abstract}
During the migration to the site of inflammation, allergic inflammatory cells must overcome several structures that constitute an impregnable barrier under normal conditions. One such structure is the extracellular matrix responsible for closely filling the intercellular space of tissues. The loosening of this tight structure by the activity of specific tissue enzymes, i.e. extracellular matrix metalloproteinases (MMPs), enables the influx of effector cells to the site of inflammation, thus facilitating the development of allergic inflammation and sustaining its presence. Specific tissue inhibitors of metalloproteinases (TIMPs) represent the most important factor involved in the regulation of MMP activity. Under physiological conditions, MMPs and TIMPs co-exist in a particular specific equilibrium, which is disturbed under pathological circumstances. The MMP-2 and MMP-9, referred to as gelatinases, belong to the best-studied MMPs associated with the development of allergic disorders. Their role pertains to the degradation of type IV collagen, the main component of basal membranes; this facilitates the influx of cells to the site of allergic inflammation.
\end{abstract}

Key words: extracellular matrix, metalloproteinases, metalloproteinase inhibitors.

\section{Introduction}

Extracellular matrix (ECM) is a complex of macromolecules such as collagen, polysaccharides, and glycoproteins, on which the cells rest. Extracellular matrix is involved in the processes of cellular growth and migration, maintenance of cellular shape, and cell-to-cell signaling. The matrix is synthesized by fibroblasts and is mostly composed of glycosaminoglycans, collagen, fibronectin, and laminin; as a result it forms the "glue" that supports the tissues and provides their stability. Under physiological conditions, ECM is constantly remodeled; the dynamic equilibrium between the formation and degradation of ECM molecules enables normal development, remodeling, and repair of tissues $[1,2]$.

In the course of an inflammatory reaction, including allergic inflammation, the tight structure of ECM is loosened due to the activity of specific tissue enzymes referred to as matrix metalloproteinases (MMPs). Consequently, various cells can infiltrate the site of ongoing inflamma- tion, enabling its maintenance and modification. Although passive degradation of ECM proteins and facilitation of cellular migration represent the principal functions of MMPs, these molecules can also constitute active modulators of inflammatory reactions, releasing growth factors, cytokines, and their receptors from intercellular space $[1,2]$. Moreover, they can be directly involved in the formation of novel receptors on the cellular surface, as well as in immunological processes via direct interaction with molecules anchored to cellular membranes [3]. Under physiological conditions, MMPs control and regulate such developmental processes as angiogenesis, embryogenesis, and tissue remodeling; moreover, they maintain homeostasis of the body and play an important role in the healing processes [4]. Furthermore, MMPs can show hyperactivity under pathological conditions, being actively involved in the pathophysiology of the latter [3].

Concentration and activity of MMPs are regulated by their natural endogenous tissue inhibitors (TIMPS). Under physiological conditions, these molecules exist in a par- 
ticular, specific equilibrium. However, this equilibrium is disturbed under pathological conditions associated with tissue destruction; attenuation of MMP hyperactivity is the main function of TIMPs under such circumstances $[2,5]$. The TIMPs are multifunctional molecules that play a variety of biological roles aside from their basic function. The TIMP-1 and TIMP-2 show the activity of factors stimulating the growth of hematopoietic progenitor cells; TIMP-1, TIMP-2, and TIMP-3 are involved in retarding the growth of neoplastic tumors, and TIMP-2 can additionally inhibit the growth of endothelial cells [1]. Moreover, TIMPs are also involved in the regulation of apoptosis: TIMP-3 shows pro-apoptotic activity, while TIMP-1 and TIMP-2 act anti-apoptotically [2]. Expression of TIMP-1 and TIMP-2 was observed on B and T lymphocytes and, furthermore, these enzymes were revealed to participate in immunological interactions, including the development of allergic response [6].

The role MMPs and TIMPs in allergic disorders is unclear. The effects of these enzymes in the pathogenesis of bronchial asthma are quite well documented, but their involvement in the development of allergic rhinitis, chronic urticaria, and atopic dermatitis was proposed quite recently. Gelatinases (MMP-2 and MMP-9) are considered the most important and best-studied MMPs involved in the development of allergic diseases; their role pertains to the degradation of type IV collagen, the main component of basal membranes. Evidence from the literature on the role of MMPs and their specific tissue inhibitors is sparse and the results of previous studies are not infrequently confusing and inconclusive, generating new questions instead of answering the ones already in existence. Taking into account the above implications, the path to understanding the role of metalloproteinases and their inhibitors in the pathogenesis of allergic conditions still seems long and requires many further, welldesigned studies.

\section{Metalloproteinases}

Metalloproteinases represent a family of extracellular or membrane endopeptidases that are active in the presence of zinc and calcium ions, in neutral or slightly alkaline environment [7]. These enzymes are released in an inactive form and become activated within the intercellular space. This process results from the removal of $10 \mathrm{kDa}$ peptide from the $\mathrm{N}$-terminal region, which exposes the zinc-containing active region [8]. The MMPs are composed of a catalytic domain and a propeptide containing a signaling peptide responsible for directing these molecules to their target sites [1].

Metalloproteinases are divided into:

- Matrilysins (endometalloproteinases): This group includes MMP-1 and MMP-7. They are characterized by the lack of a hemopexin domain. Their substrates include ECM macromolecules, as well as molecules expressed on the cellular surface such as Fas-ligand, pro-TNF, and E-cadherin. Consequently, they can participate in cell apoptosis [2].

- Collagenases (MMP-1, MMP-8, MMP-13). These molecules contain a hemopexin domain connected to the catalytic domain by a hinge region. The substrates of these enzymes include collagen types I, II, III, V, and IX [9].

- Stromelysins: this group includes two enzymes: MMP-3 and MMP-10. They play similar roles but differ in proteolytic activity. Their principal functions include hydrolysis of ECM components and activation of the inactive forms of MMPs [2].

- Gelatinases (MMP-2 and MMP-9). They are characterized by high affinity to collagen and gelatin. Additionally, MMP-2 hydrolyses peptide bonds in collagen types I, II, and III [1]. Amongst all MMPs, they are postulated to play the most significant role in allergic reactions.

- Membrane MMPs: they are divided into two groups:

- type I membrane proteins. This group includes MMP14, MMP-15, MMP-16, and MMP-24;

- GPI-anchored proteins - MMP-17 and MMP-25.

The principal function of membrane MMPs (with the exception of MMP-17) pertains to the activation of proMMP-2; their substrates include many components of ECM $[1,2]$.

\section{Metalloproteinase inhibitors}

The TIMPs are endogenous proteins, inhibitors of metalloproteinases, with a 21-29 kDa molecular mass. Their expression is controlled during the development and differentiation of tissues [1]. These proteins have two domains: C-terminal domain modulating the binding of TIMPs to MMPs and the N-terminal domain responsible for MMP inhibition [10]. To date, four inhibitors belonging to the TIMP family have been identified, ranging from TIMP-1 to TIMP-4 [1].

The principal function of TIMPs pertains to the inhibition of impaired activity of MMPs in the course of pathological processes [2]. The TIMPs can play this role due to their specific structure: their shape resembles a wedge able to enter MMP active site in a fashion similar to the substrate. TIMP binds non-covalently to a MMP molecule in a $1: 1$ ratio $[1,2]$. In this process, a specific role is played by the N-terminal domain, which chelates the atom of zinc via its amine group [7]. Additionally, the carboxyl group of $\mathrm{N}$-terminal cysteine binds to zinc, blocking the activity of the entire enzyme [2]. Aside from inhibiting active MMPs, TIMPs also block the transformation of proMMPs into the active forms, additionally inhibiting the activity of the latter [11].

\section{Matrix metalloproteinases - tissue inhibitors metalloproteinases equilibrium}

Under physiological conditions, the concentration of MMPs is regulated by their natural endogenous 
inhibitors (TIMPS), as well as by the natural non-specific protease inhibitors: $\alpha_{2}$-macroglobulin, and $\alpha_{1}$-antiprotease. Both MMPs and TIMPs are released by the same cells: monocytes, macrophages, lymphocytes, granulocytes, blood platelets, fibroblasts, keratinocytes, myocytes, neurons, astrocytes, endothelial cells, and hepatocytes, as well as by neoplastic cells $[1,11]$. Typically, TIMPs and MMPs co-exist in a particular, specific equilibrium [1]. However, the equilibrium between MMPs and TIMPs is disturbed under pathological conditions associated with tissue destruction. Although concentrations of MMPs and TIMPs are elevated in involved tissues, the increase in TIMP concentration does not fully compensate for elevated MMP activity [5].

Aside from being modulated by TIMPs, the tissue activity and concentration of MMPs depend on an array of other factors, such as inflammatory cytokines, growth factors (epidermal growth factor - EGF), hormones (steroids, parathormone - PTH), products of bacterial degradation (lipopolysaccharide - LPS), metal ions, oxidants, detergents, proteolytic enzymes, plasmin, and serpins - the inhibitors of serine proteases. Furthermore, their activity is significantly modulated by the $\mathrm{pH}$ of the intercellular environment [12].

Interleukin 1 and tumor necrosis factor $\alpha$ (TNF- $\alpha)$, as well as transforming growth factor $\beta$ (TGF- $\beta$ ), interleukin-6 (IL-6), and IL-10 are the main cytokines regulating the expression of MMPs and TIMPs. Interleukin-1 induces the activity MMPs and inhibits the synthesis of TIMP-1. The effect of TNF- $\alpha$ is similar to that of IL-1, and the two cytokines act synergistically, which potentiates their effects. In contrast, TGF- $\beta$ and steroid hormones inhibit MMPs synthesis $[6,13]$. Other factors that activate the expression of MMPs include VEGF, prostaglandins (PGE2 and PGF2 $\alpha$ ), and oncogenes. They modulate the expression of fas and jun genes, whose products form AP-1 transcription factors. These factors bind to the relevant sequences of DNA, stimulating transcription of the metalloproteinase gene [11]. Another activating cytokine, extracellular matrix metalloproteinase inducer (EMMPRIN), released by matrix cells, keratinocytes, and neoplastic cells also stimulates fibroblasts to secrete MMP [14].

Aside from endogenous mechanisms, exogenous mechanisms able to regulate MMP activity were also documented. The activation of MMPs can be induced by various inorganic compounds (cyanide, potassium iodide, dodecyl sodium sulfate), mercury-containing organic compounds (4-aminophenylmercuric acetate - APMA), urea, and oxidative stress. Conversely, factors with a documented inhibitory activity include diphosphates and tetracyclines. The mechanism of their action pertains to the chelation of calcium and zinc ions necessary for normal functioning of MMPs. Their role was confirmed by the fact that this process can be reversed by the excess of calcium and zinc ions [15]. Other inhibitors of MMPs include some of the biological agents used in the thera- py of rheumatoid arthritis, e.g. interferon- $\gamma($ IFN- $\gamma$ ) and IL-4, as well as dexamethasone and indomethacin. These compounds are effective by inhibiting the production of PGE-2 and CAMP, both participating in MMP synthesis [16].

Currently, MMPs and their endogenous inhibitors are the subject of extensive research that deals with their role in various pathological processes. To date, their role was unambiguously confirmed in such processes as the progression of neoplastic and cardiovascular disorders, Alzheimer's disease, autoimmune disorders (rheumatoid arthritis, systemic lupus erythematosus), liver cirrhosis, and Crohn's disease, as well as in the development of gastric and duodenal ulcers $[4,17,18]$.

\section{Matrix metalloproteinases and tissue inhibitors metalloproteinases in allergic disorders}

The role of MMPs and TIMPs in allergic disorders is not fully understood. This results from a small number or even lack of studies on their role in the pathogenesis of various conditions. The most important and best-studied MMPs associated with the development of allergic disorders include MMP-2 and MMP-9, referred to as gelatinases. They are principal MMPs involved in the influx of inflammatory cells to the site of allergic inflammation resulting from the degradation of type IV collagen, the main component of basal membranes. This is reflected by loosening the structure of cells and basal membranes and exposition of previously hidden collagen sites; the latter facilitate the tissue migration of inflammatory cells by binding to integrin $\alpha_{v} \beta_{3}$ located on their surface [14].

\section{Bronchial asthma}

Both MMPs and TIMPs play an active role in the airway remodeling of bronchial asthma patients. It is postulated that MMP-induced injury of ECM directly precedes airway fibrosis. The MMP-9 (gelatinase B) is the best described and most specific for bronchial asthma. It is not synthesized in healthy pulmonary tissue under physiological conditions, but can be released by many types of cells involved in the inflammatory process during exacerbation of asthma [19]. Bellegulic et al. revealed that the exacerbation of asthma is associated with an increase in serum concentration of MMP-9, while the activity of MMP-2 remains unchanged. Consequently, they suggested that MMP-9 can serve as a novel, non-invasive marker of inflammation and remodeling of bronchial tree [20]. Also, Oshita et al. observed a statistically significant increase in MMP-9 during asthma exacerbation as compared to controlled asthma; this correlated with inflammatory infiltration and bronchial remodeling. In contrast, they did not observe significant changes in MMP-2 and TIMP-1 [21]. Ko et al. reported slightly different findings: they revealed an increase in both MMP-9 and TIMP-1 in 
groups of patients with severe bronchial asthma and mucus hypersecretion as compared to a group of healthy individuals [22]. Mautino et al. observed quantitative abnormalities of MMP-9/TIMP-1 in asthma patients. The rate of collagen, proteoglycans, and elastin degradation was reduced due to the excess of TIMP-1 despite increased or normal activity of MMP-9 and was reflected by the bronchial wall accumulation of these compounds and resultant remodeling [23]. Matsumoto et al. revealed a positive correlation between an increase in TIMP-1 concentration and the thickness of the bronchial wall in bronchial asthma patients, as well as the negative correlation between MMP-9/TIMP-1 ratio and the increase in airway wall thickness and irreversible obturation [24]. A proportional relationship was observed between MMP-9/TIMP-1 ratio in the induced sputum, or FEV1 of examined patients. Higher concentrations of MMP-9 in BALF correlate with the airway influx of neutrophils in patients subjected to antigen provocation and are, to a small degree, inhibited by oral glucocorticoids. Furthermore, a positive correlation was observed between MMP-9 and TIMP-1 concentrations and the number of neutrophils in the sputum of examined subjects $[20,24]$.

Neutrophil is the most important cell secreting MMP-9 in asthma patients; the neutrophil secretion of this enzyme is approximately five-fold higher than that of eosinophils [25]. Van den Steen et al. confirmed the expression of MMP-9 molecules on the surface of activated polynuclear granulocytes. This membrane-bound form of MMP is responsible for the degradation of type IV collagen, gelatin, elastin, and $\alpha_{1}$-antiprotease inhibitor and is resistant to inactivation by TIMP. The IL- 8 activates neutrophils and stimulates them to the release MMP-9. In turn, MMP-9 modifies IL-8 molecule by splicing a sixamino acid peptide; as a result, IL-8 gains 10 - to 27 -fold stronger potential with regards to neutrophil activation, which increases the release of MMP-9 in a mechanism of positive reverse feedback [26].

\section{Atopic dermatitis}

Atopic dermatitis is a chronic inflammatory condition associated with the impairment of the epidermal barrier. Data on the role of MMPs in the inflammation associated with atopic dermatitis are still sparse. Quite recently, Harper et al. have revealed that the activity of MMPs in skin lesions associated with atopic dermatitis is approximately five-fold higher than in unaffected regions and as many as 10 to 24 times higher than in the healthy individuals. Furthermore, elevated levels of MMP-8 and MMP-9 were documented in atopic dermatitis patients, while the levels of MMP-10, TIMP-1, and TIMP-2 were markedly lower than in healthy individuals [27]. Also, Devillers et al. observed an elevated serum level of MMP-9 in atopic dermatitis patients as compared to a group of healthy indi- viduals [28]. In contrast, Katoch et al. found a higher serum level of TIMP-1, but not MMP-3, in a group of patients with exacerbated atopic dermatitis. Additionally, they observed that the level of TIMP-1 is significantly reduced following the treatment of exacerbation [29].

\section{Urticaria}

An elevated serum level of MMP-9 was also documented in chronic urticaria patients [30]. The level of MMP-9 correlated positively with symptom severity, being the highest in individuals with severe urticaria. The MMP-9 is postulated to facilitate the influx of inflammatory cells to the site of inflammation via the degradation of type IV collagen. Upon activation, these cells are able to release MMP-9 in the course of being involved in tissue injury, enhancing the vicious circle of allergic reaction [30]. Tedeschi observed an increase in both MMP-9 and TIMP-1 levels in chronic urticaria patients. Moreover, it was also noted that the level of MMP-9 correlates positively with CRP concentration, but not with the response to the administration of autologous serum and the serum level of circulating histamine-releasing factors [31]. Also, Caproni et al. observed elevated plasma levels of MMP-9 in chronic urticaria that was correlated with the degree of disease severity [32]. In contrast, Altrichter et al., based on their analysis of serum MMP-9 as the activity marker of chronic urticaria, concluded that while the level of this parameter was significantly higher as compared to the healthy controls, it did not change significantly in relation to disease severity. Furthermore, serum levels of MMP-9 in urticaria patients were comparable to those in individuals with psoriasis. The authors concluded that the serum level of MMP-9 should not be determined in the evaluation of the effectiveness of urticaria treatment and is not a useful marker of the degree of disease exacerbation [33]. An interesting study was conducted by Antiga et al. in which differences in serum levels of MMP-2, MMP-9, TIMP-1, and TIMP-2 were analyzed in patients with chronic idiopathic urticaria, autoimmune urticaria, and healthy individuals. The only significant difference pertained to MMP-9 in the group of patients with autoimmune urticaria and in those with chronic urticaria (284 ng/ml vs. 108 ng/ml). Furthermore, all individuals with autoimmune urticaria had MMP-9 levels higher than $188 \mathrm{ng} / \mathrm{ml}$ (not observed in the other groups). This suggests that serum MMP-9 can be potentially used as a specific marker for this type of urticaria [34].

\section{Allergic rhinitis}

Matrix metalloproteinases play a significant role in the etiopathogenesis of allergic conditions of nasal mucosa. Under such circumstances, the principal role is played by MMP-9, which degrades intercellular matrix in a fashion similar to other conditions. This is reflected by 
the influx of inflammatory cells. Neutrophils constitute the principal source of MMP-9 [35]. Bugdayci et al. documented elevated levels of MMP-9 in patients with nasal polyps associated with allergic rhinitis. This level was significantly higher than in individuals with nasal polyps not accompanied by allergic rhinitis [36]. Also Leonardi et al. observed that the level of MMP-9 in nasal polyp biopsies is significantly higher than in the specimens of nasal mucosa obtained from healthy individuals. Furthermore, the level of MMP-9 correlated positively with tissue eosinophilia and CD4+ lymphocyte count [37].

\section{Allergic contact dermatitis}

Presently ongoing studies are focused on the role of MMPs in allergic contact dermatitis (ACD). According to Wang et al., MMP-3 is an important factor in the initiation of ACD-associated inflammation as it induces T lymphocyte proliferation in response to a specific antigen. Furthermore, MMP-9 is responsible for maintaining skin's inflammatory response [38]. Lebre et al. revealed that MMP-9 plays a crucial role in the dermo-epidermal migration of Langerhans and dendritic cells [39]. Additionally, increased levels of MMP-2 and MMP-9 were observed in the affected skin of ACD patients; although, they were not accompanied by elevated serum activities of these enzymes [40]. Moreover, the results of a recent study by Reduta et al. suggest that TIMP-1 also plays an important role in ACD. Probably, an increased level of this enzyme reflects lower activity of skin inflammation and can, therefore, serve as a marker of remission [41].

Among others, extensive studies dealing with the role of MMPs and TIMPs in the pathogenesis of various disorders verified possible therapeutic application of specific MMP inhibitors. Such studies were undertaken in the case of allergic conditions as well. In particular, they were concentrated on bronchial asthma and analyzed the potential effect of a specific MMP inhibitor (marimastat) on the outcome of the disease. Following 3 weeks of administration of the inhibitor, no significant reduction in bronchial hyperresponsiveness to antigen challenge was observed. Moreover, the agent did not modulate the cellular profile of sputum, concentration of exhaled NO, or $\mathrm{FEV}_{1}$ [42].

\section{Conclusions}

This review of the most important studies dealing with the role of MMPs and TIMPs in the pathogenesis of allergic disorders highlights an unusual paucity of literature data with regard to the problem in question (with the exception of evidence from bronchial asthma patients). Moreover, the results of available studies are not infrequently conflictive and inconclusive and raise new questions instead of answering the ones already in existence. Finally, literature lacks any published research of the role of MMPs and TIMPs in the allergic type of food sensitivity. In view of the aforementioned implications, the path to understanding the role of metalloproteinases and their inhibitors in the pathogenesis of allergic conditions still seems long, and requires many further, welldesigned studies.

\section{References}

1. Lipka D, Boratyński J. Metalloproteinases. Structure and function. Post Hig Med Dośw 2008; 62: 328-36.

2. Visse R, Nagase H. Matrix metalloproteinases and tissue inhibitors of metalloproteinases: structure, function, and biochemistry. Circ Res 2003; 92: 827-39.

3. Hrabec E, Naduk J, Stręk M, Hrabec Z. Collagenases type IV [MMP-2 and MMP-9], and their substrates-extracellular matrix proteins, hormones, cytokines, chemokines and their receptors. Post Biochem 2007; 53: 37-45.

4. Wong T, Sethi C, Daniels J, et al. Matrix metalloproteinases in disease and repair processes in the anterior segment. Surv Ophthalmol 2002; 47: 239-56.

5. Żebrowski M, Kierus-Gudaj A, Żebrowska A. The role of metalloproteinases in etiology of coronary heart disease. Forum Kardiologów 2003; 8: 53-7.

6. Oelmann E, Herbst H, Zuhlsdorf M, et al. Tissue inhibitor of metalloproteinases 1 is an autocrine and paracrine survival factor, with additional immune-regulatory functions, expressed by Hodgkin/Reed-Sternberg cells. Blood 2002; 99: 258-67.

7. Maskos K. Crystal structures of MMPs in complex with physiological and pharmacological inhibitors. Biochimie 2005; 87: 249-63.

8. Nagase H. Activation mechanisms of matrix metalloproteinases. Biol Chem 1997; 378: 151-60.

9. Ala-aho R, Kahari V. Collagenases in cancer. Biochimie 2005; 87: 273-86.

10. Murphy G, Houbrechts A, Cockett M, et al. The N-terminal domain of tissue inhibitor of metalloproteinases retains metalloproteinase inhibitory activity. Biochemistry 1991; 30: 8097-102.

11. Kowalski M, Walczak A, Majsterek I. Matrix metalloproteinases (MMPs): modern molecular markers of open-angle glaucoma diagnosis and therapy. Postępy Hig Med Dośw 2008; 62: 582-92.

12. Birkedal $\mathrm{H}$. Role of cytokines and inflammatory mediators in tissue destruction. J Periodontal Res 1993; 28: 500-10.

13. Ruhul Amin A, Senga T, Oo M, et al. Secretion of matrix metalloproteinase- 9 by the proinflammatory cytokine, IL-1beta: a role for the dual signalling pathways. Akt Erk Genes Cells 2003; 8: 515-23.

14. Xu J, Rodriguez D, Petitclerc E, et al. Proteolitic exposure of a cryptic site within collagen type IV is required for angiogenesis and tumor growth in vivo. J Cell Biol 2001; 154: 1069-79.

15. Nip L. Inhibition of epithelial cell matrix metalloproteinases by tetracyclines. J Periodontal Res 1993; 28: 379-85.

16. Larry M, Corcoran W. Regulation of monocyte/macrophage metalloproteinase production by cytokines. J Periodontol 1993; 64: 467-73.

17. Łukaszewicz M, Mroczko B, Szmitkowski M. The role of metalloproteinases and their inhibitors in pancreatic cancer. Postep Hig Med Dośw 2008; 62: 141-7.

18. Stankiewicz-Choroszucha BL, Wawrzyniak ZM, Lipiec A, et al. Consequences of smoke inhalation in the 'Epidemiology of 
Allergic Diseases in Poland' project (ECAP). Ann Agric Environ Med 2011; 18: 420-8.

19. Fal A, Rabski M. The role of neutrophil in asthma - new therapeutic perspectives. Alergia 2006; 2: 37-42.

20. Bellegulic C, Corbel M, Germain N, et al. Increased release of matrix metalloproteinase- 9 in the plasma of acute severe asthmatic patients. Clin Exp Allergy 2002; 32: 217-23.

21. Oshita Y, Koga T, Kamimura T, et al. Increased circulating 92 kDa matrix metaloproteinase (MMP-9) in exacerbation in asthma. Thorax 2003; 58: 757-60.

22. Ko F, Diba C, Roth M, et al. A comparison of airway and serum matrix metalloproteinase-9 activity among normal subjects, asthmatic patients, and patients with asthmatic mucus hypersecretion. Chest 2005; 127: 1919-27.

23. Mautino G, Capony F, Bousquet J, et al. Balance in asthma between matrix metalloproteinases and their inhibitors. J Allergy Clin Immunol 1999; 104: 530-3.

24. Matsumoto H, Niimi A, Takemura M, et al. Relationship of airway wall thickening to an imbalance between matrix metalloproteinase-9 and its inhibitor in asthma. Thorax 2005; 60: 277-81.

25. Takafuji S, Ishida A, Miyakuni Y, et al. Matrix metalloproteinase-9 release from human leukocytes. J Investig Allergol Clin Immunol 2003; 13: 50-5.

26. Van den Steen P, Proost P, Wuyts A, et al. Neutrophil gelatinase $B$ potentiates interleukin-8 tenfold by aminoterminal processing, whereas it degrades CTAP-III, PF-4, and GROalpha and leaves RANTES and MCP-2 intact. Blood 2000; 96: 2673-81.

27. Harper J, Godwin H, Green A, et al. A study of matrix metalloproteinase expression and activity in atopic dermatitis using a novel skin wash sampling assay for functional biomarker analysis. Br J Dermatol 2010; 162: 397-403.

28. Devillers A, van Toorenenbergen A, Heerenbrink G, et al. Elevated levels of plasma matrix metalloproteinase- 9 in patients with atopic dermatitis: a pilot study. Clin Exp Dermatol 2007; 32: 311-3.

29. Katoh N, Hirano S, Suehiro M, et al. Increased levels of serum tissue inhibitor of metalloproteinase-1 but not metalloproteinase-3 in atopic dermatitis. Clin Exp Immunol 2002; 127: 283-8.

30. Kessel A, Bishara R, Amital A, et al. Increased plasma levels of matrix metalloproteinase-9 are associated with the severity of chronic urticaria. Clin Exp Allergy 2005; 35: 221-5.

31. Tedeschi A, Asero R, Lorini M, et al. Plasma levels of matrix metalloproteinase-9 in chronic utricaria patient correlate with disease severity and C-reactive protein but not with circulating histamine-releasing factors. Clin Exp Allergy 2010; 40: 875-81.

32. Caproni M, Gioni B, Volpi W. Metalloproteinase activity in chronic urticaria. EADV, $16^{\text {th }}$ Congress of the European Academy of Dermatology and Venerology. Vienna, Austria. 16-20th May 2007, P1356.

33. Altrichter S, Boodstein N, Maurer M. Matrix metalloproteinase-9: a novel biomarker for monitoring disease activity in patients with chronic utricaria patients? Allergy 2009; 64: 652-6.

34. Antiga E, Volpi W, Del Bianco E, et al. Plasma levels of metalloproteinaze-9 are elevated in patients with chronic autoimmune utricaria. Br J Dermatol 2009; 161: 712-4.

35. Rapiejko P. Early and late phase of allergic reaction of nasal mucosa. Alergoprofil 2007; 3: 7-13.

36. Bugdayci G, Kaymakci M, Bukan N. Matrix metalloproteinase-9 (MMP-9) in allergic nasal polyps. Acta Histochemica 2010; 112: 92-5.
37. Leonardi A, Brun P, Di Stefano A, et al. Matrix metalloproteinases in vernal keratoconjunctivitis, nasal polyps and allergic asthma. Clin Exp Allergy 2007; 37: 872-9.

38. Wang M, Qin J, Mudgett T. MMP deficiencies affect contact hypersensitivity: stromelysin-1 deficiency prevents the response and gelatinase $B$ deficiency prolongs the response. Immunology 1999; 96: 6885-9.

39. Lebre M, Kalinski P, Das P, et al. Inhibition of contact sensitizer-induced migration of human Langerhans cells by matrix metalloproteinase inhibitors. Arch Dermatol Res 1999; 291: 447-52.

40. Gianelli G, Foti C, Marinosci F, et al. Gelatinase expression at positive patch test reactions. Contact Dermatitis 2002; 46: 280-5.

41. Reduta T, Laudańska H, Laudanski P. Tissue inhibitors of MMP1 levels are increased in serum of patients with allergic contact dermatitis. Contact Dermatitis 2007; 57: 100-4.

42. Bruce C, Thomas P. The effect of marimastat, a metalloprotease inhibitor, on allergen-induced asthmatic hyperreactivity. Toxicol Appl Pharmacol 2005; 205: 126-32. 